\title{
Prescrição Anti-Hipertensiva e Efetividade do Controle Pressórico Ambulatorial
}

\section{Anti-Hypertensive Prescription and Effectiveness of Ambulatorial Pressure Control}

Nailton Jatobá Tenório Filho1, Rafaela Oliveira largas¹, Oliveira, L.P.S1, Danielly Dourado Santos Silva1, Gabriel Gomides Vasconcelos 1, Lorena Passos Soares ${ }^{1}$, Danival Ferreira de Castro Júnior ${ }^{1}$, Rayza Pereira Barbosa ${ }^{1}$, Marcos Renato Herrera².

\section{RESUMO}

A hipertensão arterial sistêmica é uma condição clínica multifatorial responsável direta ou indiretamente por $50 \%$ das mortes por doenças cardiovasculares. O emprego de uma terapêutica adequada reduz 0 risco de desenvolvimento de doenças cardiovasculares nesses pacientes. O objetivo deste trabalho foi avaliar a efetividade da terapia medicamentosa no controle pressórico dos pacientes assistidos no Ambulatório de cardiologia do Centro Universitário UnirG de Gurupi-To. O estudo foi do tipo retrospectivo, transversal, aprovado pelo Comitê de Ética em Pesquisa ( $n^{\circ}$ 1.907.385), utilizando como base dados de prontuários no período de 2011 a 2016. Foram selecionados 67 pacientes, destes, 76,1\% dos pacientes seguiam a terapia preconizada pela VII Diretriz de Hipertensão. Um dado preocupante foi a prevalência de pacientes que não atingiram as metas preconizadas, 46 (68,8\%). Dos pacientes que estavam dentro das metas ideais, $81 \%$ seguiam a terapêutica recomendada pela diretriz mais atual, sendo as medicações mais utilizadas os diuréticos tiazídicos e os betabloqueadores. O grau de conformidade foi considerado satisfatório. A taxa alcançada de pacientes que atingiram a meta recomendada foi semelhante aos estudos internacionais e compatível com valores encontrados em estudos brasileiros.

Palavras-chave: Hipertensão. Anti-hipertensivos. Ambulatorial.

\section{ABSTRACT}

Systemic arterial hypertension is a multifactorial clinical condition that is directly or indirectly responsible for $50 \%$ of deaths due to cardiovascular diseases. The use of appropriate therapy reduces the risk of developing cardiovascular diseases in these patients. The objective of this study was to evaluate the effectiveness of the drug therapy in the blood pressure control of patients attended at the Cardiology ambulatory of the UnirG University Center of Gurupi-TO. The study was retrospective, cross-sectional, approved by the Research Ethics Committee (No. 1,907,385), based on data from medical records in the period from 2011 to 2016 . Sixty-seven patients were selected, of which $76.1 \%$ the therapy recommended by the VII Brazilian Hypertension Guidelines. A worrying fact was the prevalence of patients who did not reach the recommended goals, $46(68.8 \%)$. About patients that were within the ideal goals, $81 \%$ followed the therapy recommended by the most current guideline, the most used medications being thiazides diuretics and beta-blockers. The degree of compliance was considered satisfactory. The achieved rate of patients that reached the recommended goal was similar to the international studies and compatible with values found in Brazilian studies.
${ }^{1}$ Acadêmicos do curso de Medicina no Centro Universitário UnirG.

E-mail:

tinho_jatoba@hotmail.com

${ }^{2}$ Docente do curso de Medicina no Centro Universitário UnirG.

Keywords: Hypertension. Antihypertensives. Ambulatorial. 


\section{INTRODUÇÄO}

A Hipertensão Arterial Sistêmica (HAS) é uma condição clínica multifatorial (Malachias et al., 2016), com alta prevalência nas populações ocidentais (NOVELLO et al., 2017) e consiste em uma das principais causas de morte em cenário mundial. No Brasil, a HAS é responsável direta ou indiretamente por $50 \%$ das mortes por doenças cardiovasculares (DCV) (Malachias et al., 2016) e estima-se que em 2025, um terço da população mundial será hipertensa (NOVELLO et al., 2017).

A diminuição do risco de DCV em pacientes com HAS diagnosticada mostrou-se satisfatória mediante uma eficaz terapêutica anti-hipertensiva (ROSENDORFF et al., 2015), tanto medicamentosa quanto não medicamentosa. Contudo, uma pesquisa americana estimou que, apesar de aproximadamente $80 \%$ dos hipertensos estarem cientes de seu diagnóstico e cerca de $75 \%$ estarem em tratamento, apenas $51 \%$ são controlados com base na diretriz do Joint National Committee-7 (KRAKOFF, 2015). No Brasil, a taxa de controle efetivo da pressão arterial (PA) está entre 10\% e 57\% (PINHO; PIERIN, 2013).

Um fator determinante no controle da HAS é a aderência ao tratamento facilitando o controle pressórico. Já os insucessos em conseguir as alcançar as metas recomendadas podem estar associados à má adesão terapêutica (LÜCHER et al, 1985; HERSHEY et al., 1980; FELDMAN et al., 1998; FLACK et al., 1996; Teresa; Karin, 2000).

A VII Diretriz Brasileira de Hipertensão Arterial (VIIDBHA) indica o uso de diuréticos, inibidores adrenérgicos, betabloqueadores, alfabloqueadores, vasodilatadores diretos, bloqueadores dos canais de cálcio, inibidores da enzima conversora de angiotensina, bloqueadores do receptor de angiotensina e inibidor direto da renina como antihipertensivos. A primeira escolha de medicamento será preferencialmente por aqueles em que haja comprovação de redução de eventos cardiovasculares, enquanto os demais ficam reservados a casos especiais em que haja a necessidade da associação a fim de que sejam atingidas as metas da PA (Silva; Petroski; Peres, 2013).

A HAS está diretamente ligada às doenças arteriais coronarianas (Moraes; Checchio; Freitas, 2015; Moscavitch et al., 2009) e tem como consequência efeitos deletérios sistêmicos. Em estudos brasileiros, grande parte do conhecimento e publicações sobre prevalência, distribuição e fatores associadas está predominantemente localizado em capitais ou Regiões Metropolitanas do Sul e Sudeste, sendo escassos ou inexistentes estudos epidemiológicos situados em municípios da Região Norte, principalmente em cidades interioranas (Moscavitch et al., 2009). 
Este trabalho teve por objetivo avaliar os prontuários de atendimentos, discorrendo sobre a conformidade com a VIIDBHA, a efetividade da terapia medicamentosa no controle pressórico correlacionando com outros estudos semelhantes, assim como, as dificuldades encontradas pelos médicos durante esse tratamento.

\section{MATERIAIS E METODOS}

O estudo é do tipo descritivo, retrospectivo, transversal. Os dados utilizados foram coletados nos prontuários da especialidade de cardiologia no ambulatório da UnirG em Gurupi, estado do Tocantins, no período de 2011 a 2016, sendo utilizada somente a última consulta de cada paciente para o presente estudo.

Foram avaliados todos os pacientes hipertensos em tratamento que foram atendidos no ambulatório da UnirG na especialidade de Cardiologia no período de 2011 a 2016, os registros nos prontuários seguem as recomendações do Ministério da Saúde. Foram incluídos pacientes hipertensos de todas as faixas etárias, sexo, etnias, período de evolução da doença ou condições socioeconômicas. Foram excluídos todos os participantes que não satisfizeram os critérios de inclusão e os prontuários que impossibilitaram a coleta de dados.

Nas consultas, todos os pacientes foram submetidos a anamnese, exame físico com antropometria, aferição de PA em dois momentos diferentes na posição ortostática e duas aferições supinas em ambos os membros, sendo registrado o maior valor. Para a aferição da PA foram respeitadas as recomendações da Sociedade Brasileira de Cardiologia. A conduta foi realizada por médicos especialistas na área (cardiologistas).

A coleta de dados foi realizada no próprio ambulatório, em sala a parte, em dias estipulados pela Secretaria Municipal de Saúde de Gurupi - TO. Os dados coletados foram transcritos para o programa Microsoft Excel 2016 onde foi possível quantificar sexo, PA, comorbidades e medicamentos em uso. A partir de tais dados pudemos descrever a epidemiologia, terapêutica vigente e se tal terapêutica alcançou a meta definida pela VIIDBHA.

A classificação de HAS seguiu o recomendado pela VIIDBHA, que estabelece como metas: medidas < 140/90 para HAS grau I e II com baixo risco cardiovascular e HAS Grau III e $<130 / 80$ para HAS grau I e II de moderado e alto risco. Considerou-se a efetividade da terapia medicamentosa, segundo o recomendado pela diretriz, no alcance das metas recomendadas. 
Foi considerado condizente com a terapêutica recomendada diretriz os pacientes em uso de diuréticos tiazídicos (DT), Inibidor da Enzima Conversora da Angiotensina (IECA) ou Bloqueador do Receptor de Angiotensina (BRA), Bloqueador do Canal de Cálcio (BCC) e Betabloqueador (BB), assim como suas respectivas associações.

\section{CONSIDERAÇÕES ÉTICAS}

Esta pesquisa se respalda na resolução ํo. 466/2012 do Conselho Nacional de Saúde que regula as pesquisas com seres humanos e foi aprovada pelo Comitê de Ética em Pesquisa do Centro Universitário UNIRG, por meio do parecer com protocolo № 1.907.385 e autorizada pela Coordenação de Gestão da Educação na Saúde - CGES, departamento da Secretaria Estadual de Saúde que autoriza a realização de pesquisas em unidades de saúde do estado do Tocantins.

A captação dos dados foi feita no Ambulatório de Saúde Comunitária da UnirG pelos membros da Liga do Coração - LCor, em uma sala separada, em três manhãs na semana, segundo orientação da Secretaria de Saúde do Município.

\section{RESULTADOS}

Foram analisados 127 prontuários do Ambulatório de Cardiologia do Curso de Medicina do Centro Universitário UNIRG, desses foram excluídos prontuários incompletos e selecionados 67 pacientes que atendiam aos critérios de inclusão. Para uma maior eficiência e igualdade entre os diferentes participantes foram analisadas somente as últimas consultas de cada paciente. Dos 67 pacientes hipertensos participantes da pesquisa, $52,2 \%$ pertencem ao sexo feminino. A média de idade foi de 57,9 anos e desvio padrão de 16,4 anos (Tabela 1).

Em nosso estudo 21 pacientes $(31,4 \%)$ dos pacientes atingiram as metas preconizadas pela VIIDBHA, enquanto 46 pacientes $(68,8 \%$ ) estão fora dela (Tabela 2 ).

Tabela 1. Perfil sociodemográfico dos pacientes

\begin{tabular}{lcc}
\hline \multicolumn{1}{c}{ Característica } & Valor & $\%$ \\
\hline Sexo & & \\
$\quad$ Masculino & 32 & 47,8 \\
Feminino & 35 & 52,2 \\
& & \\
Idade (Média em anos +_desvio padrão) & $57,9+\ldots 16,4$ & \\
\hline
\end{tabular}

Nota: $\%=$ porcentagem 
Tabela 2. Pacientes que atingiram ou não a meta

\begin{tabular}{lccc}
\hline & Característica & Valor & $\%$ \\
\hline Atingiram a meta & 21 & 31,4 \\
Não atingiram a meta & 46 & 68,6 \\
\hline Total & 67 & 100
\end{tabular}

Nota: $\%=$ porcentagem.

Do total de pacientes, 50,7\% tinham como meta PA $<130 / 80$ e $49,2 \%$ tinham como meta $P A<140 / 90$ (Tabela 3). Dos pacientes que atingiram a meta $66,6 \%$ tinham como meta PA $<130 / 80 \mathrm{mmHg}$ e $33,3 \%$ tinham como meta uma PA $<140 / 90 \mathrm{mmHg}$ (Tabela 4).

Tabela 3. Pacientes com meta para $<130 / 80 \mathrm{mmHg}$ e $<140 / 90 \mathrm{mmHg}$

\begin{tabular}{lll}
\hline Característica & Valor & $\%$ \\
\hline$<130 / 80 \mathrm{mmHg}$ & 33 & 50,7 \\
$<140 / 90 \mathrm{mmHg}$ & 34 & 49,2 \\
\hline Total & 67 & 100 \\
\hline
\end{tabular}

Nota: \% = porcentagem.

Tabela 4. Pacientes que atingiram a meta para $<130 / 80 \mathrm{mmHg}$ e $<140 / 90 \mathrm{mmHg}$

\begin{tabular}{lll}
\hline Característica & Valor & $\%$ \\
\hline$<130 / 80 \mathrm{mmHg}$ & 14 & 66,6 \\
$<140 / 90 \mathrm{mmHg}$ & 7 & 33,3 \\
\hline Total & 21 & 100
\end{tabular}

Nota: $\%=$ porcentagem

Obtivemos que $76,1 \%$ dos pacientes seguiam a terapia preconizada pela VIIDBHA (tratamento não medicamentoso e medicamentoso) sendo que os medicamentos de escolha são combinações entre os anti-hipertensivos, que consiste nas classes: DT, BRA, IECA e BCC e BB. Já nos demais pacientes, obtivemos $21,9 \%$ nos quais a conduta não seguiu a diretriz (Tabela 5).

Dos pacientes em terapia medicamentosa, $63,6 \%$ conseguiram atingir a meta e $36,4 \%$ não conseguiram. Os medicamentos mais utilizados foram os DT $(53,7 \%)$ e BRA $(47,7 \%)$ e a associação mais encontrada foi DT e BRA $(11,9 \%)$. 
Tabela 5. Perfil farmacológico geral dos pacientes em tratamento

\begin{tabular}{lll}
\hline Característica & Valor & $\%$ \\
\hline Seguem a diretriz & 42 & 59,7 \\
Tratamento não medicamentoso & 11 & 16,4 \\
Não seguem a diretriz & 16 & 21,9 \\
\hline Total & 67 & 100 \\
\hline
\end{tabular}

Nota: $\%=$ porcentagem

Gráfico 1. Perfil farmacológico geral dos pacientes em tratamento

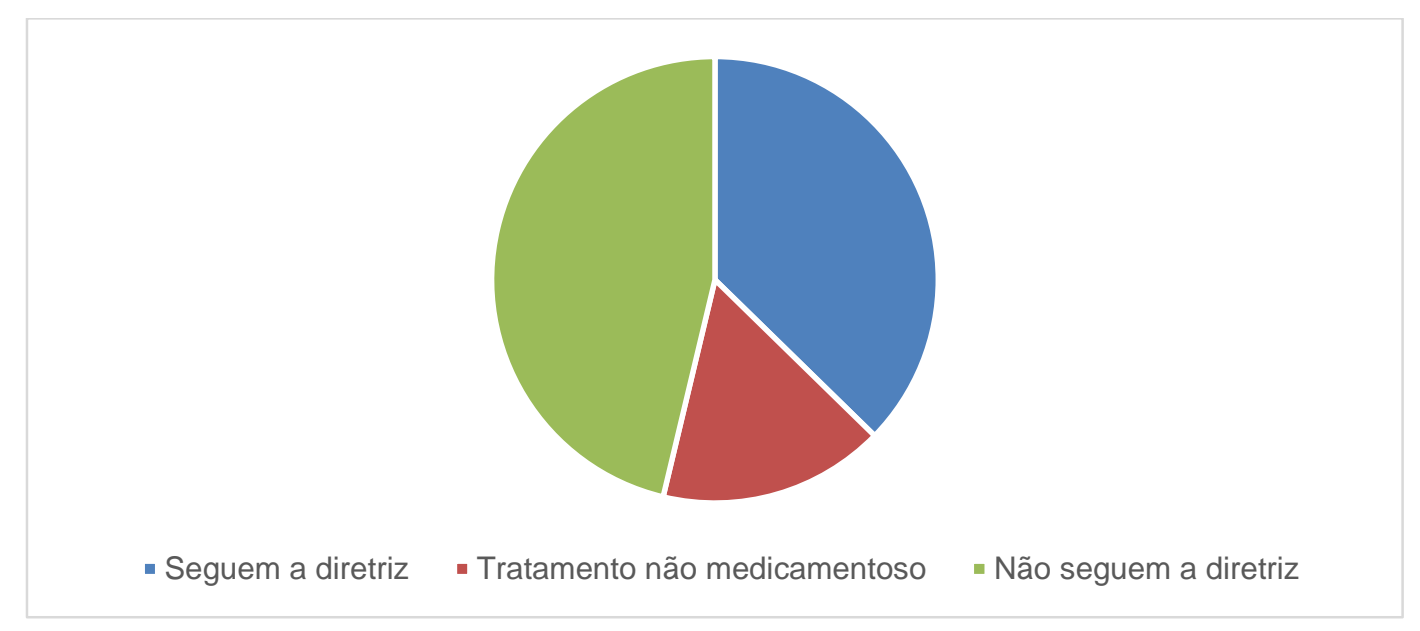

Dos tratamentos iniciados para os pacientes que atingiram a meta, 33,3\% seguiram o tratamento não medicamentoso e $66,6 \%$ seguiram o tratamento medicamentoso. 0 tratamento mais eficiente foi o não medicamentoso baseado na Mudança do Estilo de Vida (MEV), sendo 33,3\% dos pacientes que atingiram a meta somente com esse tratamento. Destes, $81 \%$ estavam em conformidade com as recomendações. As terapias de maior eficiência foram baseadas na associação de DT, BB, BCC e BRA que corresponde a 9,5\% dos pacientes que atingiram meta e o uso da monoterapia com BB que também atingiu a meta em $9,5 \%$ dos pacientes. As outras associações não pré-citadas correspondem a 4,7\% dos pacientes que atingiram a meta, cada (Tabela 6 ).

Os anti-hipertensivos mais usados em pacientes que atingiram a meta foram os DT (8) e BB (8), seguidos de BRA (6), BCC (5), IECA (3) e Espironolactona (3), e Alfa Bloqueador (1) (Gráfico 2). 
Tabela 6. Medicamentos usados pelos pacientes que atingiram a meta

\begin{tabular}{lccc}
\hline \multicolumn{1}{c}{ Característica } & Valor & $\%$ \\
\hline Alfa Bloqueador & 1 & 4,7 \\
Diurético Tiazídico + Betabloqueador + Espirinolactona + BRA & 1 & 4,7 \\
Betabloqueador + Bloqueador do Canal de Cálcio & 1 & 4,7 \\
Diurético Tiazídico + Betabloqueador + Bloqueador do Canal de Cálcio + BRA & 2 & 9,5 \\
Diurético Tiazídico + Bloqueador do Canal de Cálcio & 1 & 4,7 \\
IECA & 1 & 4,7 \\
Betabloqueador & 2 & 9,5 \\
Diurético Tiazídico + BRA & 1 & 4,7 \\
Diurético Tiazídico + Betabloqueador + IECA + BRA & 1 & 4,7 \\
Diurético Tiazídico + Bloqueador do Canal de Cálcio + IECA & 1 & 4,7 \\
Espironolactona + Betabloqueador + BRA & 1 & 4,7 \\
Diurético Tiazídico + Espironolactona & 1 & 4,7 \\
Não estavam em de anti-hipertensivos & 7 & 33,3 \\
\hline Total & 21 & 100
\end{tabular}

Nota: \% = porcentagem; IECA = Inibidor da Enzima de Conversão da Angiotensina; BRA = Bloqueador do Receptor AT1 da Angiotensina.

Gráfico 2 - Distribuição gráfica dos medicamentos usados pelos pacientes que atingiram a meta

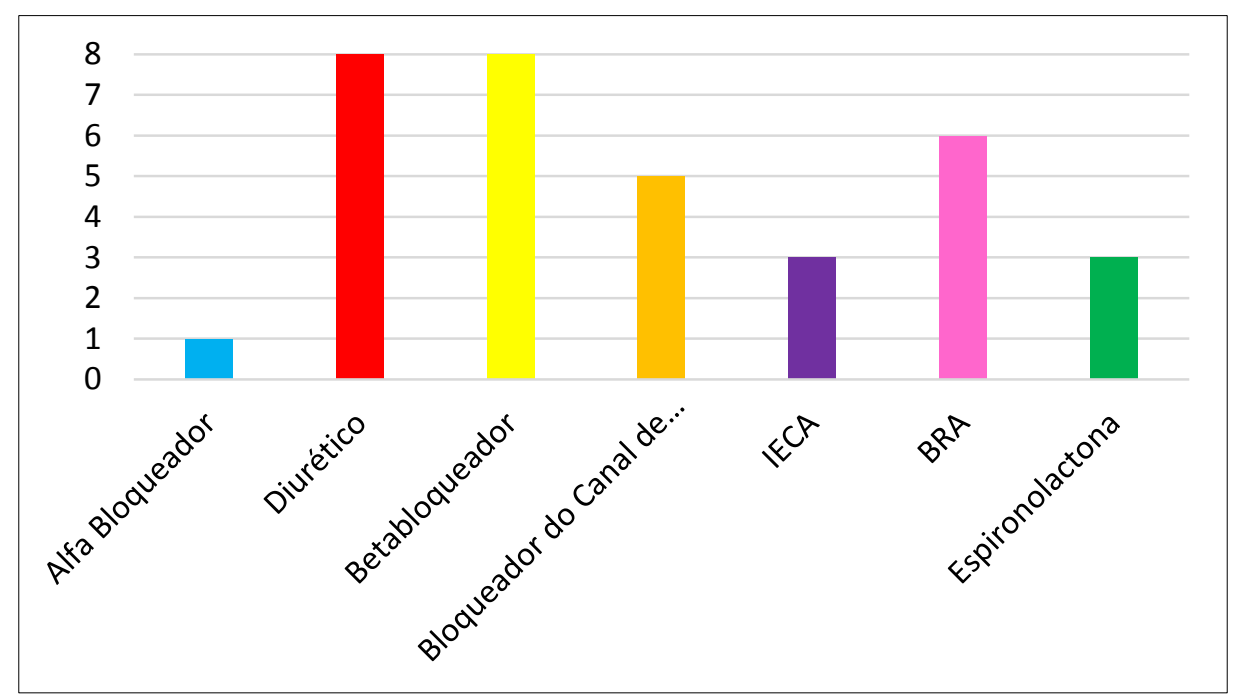

Nota: IECA = Inibidor da Enzima de Conversão da Angiotensina; BRA = Bloqueador do Receptor AT1 da Angiotensina.

\section{DISCUSSAO}

Neste estudo, observou-se que a taxa de adequação das prescrições médicas baseadas nas recomendações da VIIDBHA (MALACHIAS et al., 2016) foi de 76,1\%. 
Semelhante a outro estudo conduzido entre Agosto de 2011 a Novembro de 2012 em Niterói-RJ, no qual a taxa de adequação foi de 80\% (NOVELLO et al., 2017).

Os medicamentos mais usados foram os DTs, prescrito em 53,4\% dos casos, em conformidade com diversos estudos que avaliaram as medicações anti-hipertensivas mais utilizadas no Brasil (SANTOS JR et al., 2011; AKASHI et al., 1998). Essa classe de medicamento também foi, em conjunto com os BB, os mais usados em pacientes que atingiram meta, presente em 38,1\% das prescrições. O segundo mais utilizado foi BRA $(47,7 \%)$, contrapondo outros estudos que demonstraram preferência pela prescrição de IECA em unidades públicas de saúde (OLIVERA et al., 2010; LINARELLI et al., 2009). A associação mais frequente foi entre DT e BRA $(11,9 \%)$, diferentemente da literatura atual em que a associação mais frequente foi de diuréticos com IECA ${ }^{18}$. A preferência pelo uso do BRA em detrimento ao uso do IECA pode ser justificada por este último apresentar mais efeitos adversos (MALACHIAS et al., 2016). São incomuns os efeitos adversos relacionados aos BRA, sendo o exantema raramente observado. Pelas mesmas razões dos IECA, são contraindicados na gravidez, devendo os mesmos cuidados serem tomados em mulheres em idade fértil (MALACHIAS et al., 2016).

Foi observado que a espironolactona esteve em uso em $19,4 \%$ de todos os pacientes e em $81,2 \%$ daqueles que não seguiam a VIIDBHA. O uso desta se justificou pela associação a outras comorbidades e HAS refratária no ambulatório de cardiologia. Outro ponto questionável encontrado foi uso de alfa-bloqueador em monoterapia, já que este medicamento tem efeito hipotensor discreto sendo recomendado seu uso em associações (BRASIL, 2007).

Apesar de muitos autores publicarem que os BB não deveriam ser utilizados como anti-hipertensivos de primeira linha (MATSUSHITA et al, 2010), nosso estudo mostrou que $38 \%$ dos pacientes que atingiram meta estavam em uso desta medicação.

Observou-se a associação não recomendada de IECA com BRA em 3\% dos pacientes. Esta associação atualmente não é aceita (PÓVOA et al., 2014) pois, além de não mostrar benefício em desfecho cardiovascular, acrescenta risco de efeitos adversos (MACEDO et al., 2005).

Com relação às metas, no presente estudo 21 (31,3\%) pacientes atingiram as metas preconizadas ao contrário dos demais 46 (68,8\%). Por outro lado, um estudo com 5.023 adultos em 2003 em Portugal revelou controle pressórico em apenas 11,2\% dos pacientes (YUSUF et al, 2008), já um estudo com mais de 120.000 pacientes entre 2005 e 2011 na Itália evidenciou uma taxa de controle de 33,6\% (TOCCI et al., 2015). No Canadá em 2009, 
o percentual de hipertensos com $\mathrm{PA}<140 / 90 \mathrm{mmHg}$ foi de 64,6\% (MCALISTER et al., 2011).

Dos pacientes que atingiram a meta, 66,6\% tinham como objetivo uma PA <130/80 $\mathrm{mmHg}$ e 33,3\% PA <140/90 mmHg. Alguns pacientes que se apresentavam em estágio III estavam sem terapia otimizada, sugerindo que o médico é, muitas vezes, cauteloso demais para intensificar o tratamento anti-hipertensivo nesses pacientes, possivelmente pelo receio de efeitos adversos. Outras justificativas para esse dado é que o controle pressórico para PA <140/90 é mais difícil em idosos, sendo eles grande parte de nosso estudo ou também erros de conduta médica, má aderência à terapia (LÜCHER et al., 1985; HERSHEY et al., 1980; FELDMAN et al., 1998; FLACK et al., 1996; TERESA; KARIN, 2000). Sendo assim, uma boa orientação, adequada conhecimento médica sobre os consensos, adesão ao tratamento são fundamentais para o sucesso terapêutico.

Revelou-se que $16,4 \%$ dos pacientes estavam em tratamento não medicamentoso, esse número se torna maior quando analisamos somente os pacientes que atingiram meta chegando a 33,3\%, evidenciando assim, uma abordagem eficaz no tratamento de HAS leve.

A VIIDBHA (MALACHIAS et al., 2016) traz que o controle de peso é o tratamento não medicamentoso mais eficaz (LOPES; MORAES, 2017) responsável por uma redução de $20 \%$ a $30 \%$ da PA a cada 5\% de perda ponderal (DEMARCO; AROOR; SOWERS, 2014), já a adoção da dieta DASH pode reduzir a PA em 6,7/3,5 mmHg (SANEEI, 2014). Outra recomendação não medicamentosa eficaz seria a prática de exercício físico aeróbico capaz de reduzir a PA em 8,3/5,2 mmHg (DUNN et al., 1999), sendo esse o tratamento não medicamentoso de mais fácil adesão (OLIVEIRA, 2013). No presente estudo, não foram avaliados quais tipos de prescrições não medicamentosas foram feitas, sendo somente relatado o que foi prescrito.

É importante ressaltar que este trabalho teve como intuito comparar o tratamento prescrito com o controle pressórico, porem foi difícil estabelecer uma relação direta entre a prescrição adequada e o controle da PA, devido ao controle pressórico ser resultado da interação de diversos fatores genéticos, metabólicos e ambientais (MALACHIAS, 2016).

Além disso, foi observado um fator limitante em nosso estudo que foram as anotações incompletas em prontuários por parte dos médicos, limitando o número adequado de prontuários que foram analisados.

Novas pesquisas devem ser realizadas na intenção de obter dados que reforcem os resultados encontrados neste e em outros estudos de caráter ambulatorial para subsidiar as condutas empregadas neste meio de tratamento clínico. 


\section{CONSIDERAÇOEES FINAIS}

O grau de conformidade com a VIIDBHA foi considerado satisfatório. A taxa alcançada de pacientes que atingiram a meta recomendada foi semelhante aos estudos internacionais e compatível com valores encontrados em estudos brasileiros. Todavia, a presença de comorbidades associadas à hipertensão, aliado à idade avançada dos pacientes torna o controle pressórico ambulatorial um desafio. Dessa forma, a prescrição deve ser individualizada, tendo como base não só a HAS, como também as comorbidades associadas e condições socioeconômicas dos pacientes ambulatoriais.

\section{REFERÉNCIAS}

AKASHI, D. et al. [Antihypertensive treatment. Prescription and cost of drugs. Survey in a tertiary care hospital]. Arq Bras Cardiol., n. 71, a. 1, p. 55-7, 1998.

BRASIL, Ministério da Saúde. Wannmacher, Lenita. "Rediscutindo o uso de betabloqueadores na hipertensão." Uso racional de medicamentos: temas selecionados. Brasília: Ministério da Saúde (2007).

DEMARCO, V. G.; AROOR, A. R.; SOWERS, J. R. The pathophysiology of hypertension in patients with obesity. Nat Rev Endocrinol., n. 10, a. 6, p. 364-76, 2014.

DUNN, A. L. et al. Comparison of lifestyle and structured interventions to increase physical activity and cardiorespiratory fitness: a randomized trial. JAMA, n. 281, a. 4, p. 327-34, 1999.

FELDMAN, R. et al. Adherence to pharmacologic management of hypertension. Can $\mathbf{J}$ Public Health, n. 89, p. 116-8, 1998.

FLACK, J. M. et al. Benefits of adherence to anti-hypertensive drug therapy. Eur Heart $\mathbf{J}$, n. 17 (Suppl. A), p. 16-20, 1996.

HERSHEY, J. C. et al. Patient compliance with antihypertensive medication. Am J Public Health, n. 70, p. 1081-9, 1980.

KRAKOFF, Lawrence R. Modern Hypertension. Managemen, n. 66, p. 11061107, originally published nov./ 2015.

LINARELLI, M. C. et al. Análise do uso racional de medicamentos anti-hipertensivos utilizados em hospital-escola. Rev Ciênc Méd., n. 18, a. 4, p. 193-200, 2009.

\section{LOPES, L. O.; MORAES, E. D. Tratamento não medicamentoso para a hipertensão} arterial. Disponível em: <https://www.inesul.edu.br/revista_saude/arquivos/arqidvol_10_1339682941.pdf>. Acesso em: 02 ago. 2017.

LÜCHER, T. F. et al. Compliance in hypertension: facts and concepts. J Hypertens, n. 3, p. 3-9, 1985. 
LUPPEN, L. S. et al. Prevalence of use of antihypertensive drugs in patients followed in a Basic Health Unit of Canoas, RS, Brazil. Rev AMRIGS, n. 55, a. 1, p. 42-7, 2011.

MACEDO, M. E. et al. Prevalence, awareness, treatment and control of hypertension in Portugal: the PAP study. J Hypertens, n. 23, a. 9, p. 1661-6, 2005.

MALACHIAS, M. V. B. et al. 7aㅡ Diretriz Brasileira de Hipertensão Arterial. Arq. Bras. Cardiol, São Paulo, n. 107 (Supl. 3), p. 25, 35, 36, 2016.

MATSUSHITA, K. et al. Chronic Kidney Disease Prognosis Consortium. Association of estimated glomerular filtration rate and albuminuria with all-cause and cardiovascular mortality in general population cohorts: a collaborative meta-analysis. Lancet, n. 375, p. 2073-81, 2010.

MCALISTER, F. A. et al. Changes in the rates of awareness, treatment and control of hypertension in Canada over the past two decades. CMAJ, n. 183, a. 9, p. 1007-13, 2011.

MORAES, S. A.; CHECCHIO, M. V.; FREITAS, I. C. M. O efeito independente da obesidade central sobre a hipertensão arterial em adultos residentes em Ribeirão Preto, SP, 2007. Projeto EPIDCV. Rev Bras Epidemiol, n. 18, a. I, p. 157-73, 2015.

MOSCAVITCH, S. D. et al. Are the heart failure guidelines being implemented in primary care? Rev Port Cardiol., n. 28, a. 6, p. 683-96, 2009.

NOVELLO, Mayra Faria et al. Conformidade da Prescrição Anti-Hipertensiva e Controle da Pressão Arterial na Atenção Básica. Arq. Bras. Cardiol., São Paulo, v. 108, n. 2, p. 135142, fev./2017.

OLIVEIRA, T. L. Eficácia da educação em saúde no tratamento não medicamentoso da hipertensão arterial. Acta Paul Enferm., n. 26, a. 2, p. 179-84, 2013.

OLIVERA, C. M. et al. Prescription patterns of angiotensin converting enzyme inibhitors for Unified Health System users. Rev Bras Cardiol., n. 23, a. 3, p. 171-7, 2010.

PINHO, Natália de Alencar; PIERIN, Angela Maria Geraldo. O controle da hipertensão arterial em publicações brasileiras. Arq. Bras. Cardiol., São Paulo, v. 101, n. 3, p. e65-e73, set./2013.

PÓVOA, R. et al. I brazilian position paper on antihypertensive drug combination. Arq Bras Cardiol., n. 102, a. 3, p. 203-10, 2014.

ROSENDORFF, Clive et al. Treatment of Hypertension in Patients with Coronary Artery Disease. A Case-Based Summary of the 2015 AHA/ACC/ASH Scientific Statement. Am J Med., n. 129, a. 4, p. 372-8, 2015.

SANEEI, P. Influence of Dietary Approaches to Stop Hypertension (DASH) diet on blood pressure: a systematic review and meta-analysis on randomized controlled trials. Nutr Metab Cardiovasc Dis., n. 24, a. 12, p. 1253-61, 2014. 
SANTOS JR, E. B. et al. Perfil da Terapêutica anti-hipertensiva em uma Unidade Básica de Saúde no município de Santa Cruz-RN. Biofar, n. 5, a. 2, p. 107-15, 2011.

SILVA, D. A.; PETROSKI, E. L.; PERES, M. A. Accuracy and measures of association of anthropometric indexes of obesity to identify the presence of hypertension in adults: a population-based study in Southern Brazil. Eur J Nutr, n. 52, a. 1, p. 237-46, 2013.

TERESA, M. Z.; KARIN, S. C. Hypertension and current issues in compliance and patients outcome. Current Hypertension Reports, n. 2, p. 510-4, 2000.

TOCCI, G. et al. Blood pressure levels and control in Italy: comprehensive analysis of clinical data from 2000-2005 and 2005-2011 hypertension surveys. J Hum Hypertens, n. 29, a. 11, p. 696-701, 2015.

YUSUF, S. et al. ONTARGET Investigators. Telmisartan, ramipril, or both in patients at high risk for vascular events. N Engl J Med., n. 358, a. 15, pp. 1547-59, 2008. 